\title{
Misregulation of the arginase pathway in tissues of spontaneously hypertensive rats
}

\author{
Teddy Bagnost ${ }^{1,2}$, Alain Berthelot ${ }^{1}$, Magalie Alvergnas ${ }^{1}$, Carole Miguet-Alfonsi ${ }^{1}$, Claire André ${ }^{2}$, \\ Yves Guillaume ${ }^{2}$ and Céline Demougeot ${ }^{1}$
}

There is a growing evidence that arginase has a role in the pathophysiology of cardiovascular diseases including hypertension. We recently reported arginase upregulation in aortas from hypertensive spontaneously hypertensive rats (SHRs). The aim of this study was to determine whether arginase abnormalities occur in other tissues of SHR, including the target organs of hypertension. Experiments were conducted on 5-, 10-, 19- and 26-week-old SHRs and Wistar-Kyoto (WKY) rats. Arginase activity and expression were evaluated in heart, kidney, liver, lung and brain tissue extracts. To investigate the role of blood pressure by itself in arginase abnormalities, arginase activity was determined in 10-week-old SHRs previously treated with hydralazine ( $20 \mathrm{mg} \mathrm{kg}^{-1}$ per day, for 5 weeks). Compared with WKY rats, cardiac arginase activity was higher in hypertensive SHRs aged 10 weeks $(+46 \%, P<0.05), 19$ weeks $(+29 \%, P<0.05)$ and 26 weeks $(+23 \%, N S)$. Similar results were found in lungs in which arginase activity was increased in SHRs aged 10 weeks $(+39 \%, P<0.05), 19$ weeks $(+49 \%, P<0.05)$ and 26 weeks $(+36 \%, P<0.05)$ compared with WKY rats. The changes in arginase activity in these tissues were not associated with changes in enzyme expression. The prevention of hypertension by hydralazine blunted the increase in arginase activity in the hearts but not in the lungs. No change in arginase activity/expression was found in the kidney, liver or brain. In conclusion, this study shows that increased arginase activity is not restricted to large vessels in SHRs and suggests that cardiac arginase activity is hemodynamic sensitive.

Hypertension Research (2009) 32, 1130-1135; doi:10.1038/hr.2009.153; published online 18 September 2009

Keywords: arginase; heart; lungs

\section{INTRODUCTION}

Arginase (L-arginine ureohydrolase, EC 3.4.3.1) catalyzes the hydrolysis of arginine to ornithine and urea. Two arginase isoenzymes exist (arginase I and II), which differ in subcellular localization, regulation and possibly function. ${ }^{1}$ Arginase I is a cytosolic enzyme, which is expressed mainly in the liver as a part of the urea cycle, whereas arginase II is a mitochondrial protein that is highly expressed in the kidney and to a lesser extent in many other tissues. As extrahepatic tissues do not possess a complete urea cycle, the biological function of arginase in these tissues is presently unclear. Recent evidence showed that arginase is capable of reciprocally regulating nitric oxide (NO) synthesis by preferentially using the common NO synthase substrate Larginine. $^{2-6}$ The demonstration that both arginase isoforms are expressed by vascular endothelial and smooth muscle cells explains the growing interest in this enzyme in cardiovascular physiology and pathology. ${ }^{2-6}$

Although decreased NO bioavailability-referred to as endothelial dysfunction - has been largely implicated in the pathogenesis and pathophysiology of hypertension, ${ }^{7}$ the role of arginase in hypertension has been poorly investigated. A few data reported that vascular arginase activity and/or expression was upregulated in models of secondary hypertension, ${ }^{5,8,9}$ as well as in genetic hypertension. ${ }^{10,11}$ In these studies, isolated vessels incubated with an arginase inhibitor have been shown to recover normal NO production. From these data the new concept that targeting vascular arginase could be a novel therapeutic strategy against endothelial dysfunction in hypertension has emerged. In accordance with this hypothesis, we recently showed that in vivo pharmacological blockade of arginase decreased endothelial dysfunction and prevented blood pressure rising in prehypertensive spontaneously hypertensive rats (SHRs) and young adult SHRs. ${ }^{10,12}$

These latter studies only focused interest on the role of arginase in aortic and mesenteric endothelial dysfunction in SHRs, and as yet no study investigated the arginase pathway in tissues that encompass those implicated in the pathogenesis and pathophysiology of genetic hypertension, such as the heart, brain and kidney. However, hypertension-related damage to these target organs is a major cause of

${ }^{1}$ Laboratoire de Physiologie_-Pharmacologie—Nutrition Préventive Expérimentale, Besancon cedex, France and ${ }^{2}$ Laboratoire de Chimie Analytique, Equipe Sciences Séparatives, Biologiques et Pharmaceutiques, EA 4267, Faculté de Médecine-Pharmacie, Besancon cedex, France

Correspondence: Dr C Demougeot, Laboratoire de Physiologie-Pharmacologie-Nutrition Préventive Expérimentale, EA 4267 Faculté de Médecine-Pharmacie, Place Saint-Jacques, Besancon cedex 25030, France.

E-mail: cdemouge@univ-fcomte.fr

Received 20 February 2009; revised 25 August 2009; accepted 25 August 2009; published online 18 September 2009 
morbidity and mortality, and future antihypertensive therapy is expected to prevent or reduce it. Considering that arginase might be a valuable new target for antihypertensive therapy, it is important to determine whether the arginase pathway is impaired in organs from hypertensive animals.

In this study, we investigated arginase expression/activity in the heart, kidney, brain, lung and liver of SHRs, from prehypertensive to established stages of hypertension, and in age-matched control Wistar-Kyoto (WKY) rats. In addition, we determined whether blood pressure rising by itself accounts for the misregulation of the arginase pathway.

\section{METHODS}

\section{Animals}

In this study, 32 male WKY rats and 48 male SHRs from Charles River (L'Arbresle, France) were divided into four age groups corresponding to different phases of the development of hypertension: 5- (onset), 10- (rapid development), 19- and 26-weeks old (plateau phase). Animals were kept under a $12-12 \mathrm{~h}$ light-dark cycle and allowed free access to food and water. The investigation conforms to the Guide for the Care and Use of Laboratory Animals published by the US National Institutes of Health (NIH publication No. 85-23, revised 1996).

\section{Tissue preparation and morphological analysis}

After anesthesia with sodium pentobarbital $\left(60 \mathrm{mg} \mathrm{kg}^{-1}\right.$, i.p. $)$, the brain, kidneys, liver, lungs and heart were removed, cleaned and promptly frozen in liquid nitrogen and stored at $-80^{\circ} \mathrm{C}$ until they were processed. Heart and kidney mass indexes were calculated from their respective weights normalized for body weight.

\section{Arterial blood pressure recording}

Systolic blood pressure (SBP) was measured in warmed, restrained, conscious rats by the indirect plethysmographic tail cuff method using an automatic BP monitoring system (BP 2000, Visitech System, Apex, NC, USA).

\section{Arginase activity assay}

Arginase activity was determined in organs from urea production using the spectrophotometric method of Corraliza et al. ${ }^{13}$ as previously described in detail..$^{10}$ The amount of urea production was calculated from a standard curve and was expressed as pmol urea $\mathrm{min}^{-1}$ per mg protein.

\section{Arginase expression}

After homogenization in ice-cold lysis buffer (phosphate-buffered saline containing $1 \%$ SDS, $2 \mathrm{mmoll}^{-1}$ ethylenediamine tetraacetic acid, $2 \mathrm{mmoll}^{-1}$ ethyleneglycol bisaminoethyl tetraacetic acid, $1 \mathrm{mmoll}^{-1}$ phenylmethylsulfonyl fluoride, $2 \mu \mathrm{g} \mathrm{ml}^{-1}$ aprotinin and leupeptin), tissues were sonicated and centrifuged at $10000 \times \mathrm{g}$ for $10 \mathrm{~min}$. An aliquot of the supernatant was kept for protein measurement by the Lowry method. ${ }^{14}$ Equal amounts of total proteins $(80 \mu \mathrm{g}$ per lane for liver and kidney; $150 \mu \mathrm{g}$ per lane for brain, left ventricle and lung) were dissolved in $62.5 \mathrm{mmoll}^{-1}$ Tris- $\mathrm{HCl}(\mathrm{pH} 6.8), 2 \%$ SDS, $4 \%$ glycerol, $0.01 \%$ bromophenol blue and $10 \% \beta$-mercaptoethanol, and were heated at $95^{\circ} \mathrm{C}$ for $10 \mathrm{~min}$. Rat liver homogenate was used as a positive control for arginase I and rat kidney as a positive control for arginase II. Proteins were separated on $10 \%$ SDS-polyacrylamide gel electrophoresis according to the Laemmli method. ${ }^{15}$ Proteins were electrophoretically transferred to a PVDF (polyvinylidene fluoride) membrane $(0.2 \mu \mathrm{m}$ pore size) in cold transfer buffer ( $50 \mathrm{mmoll}^{-1}$ Tris-HCl, $50 \mathrm{mmoll}^{-1}$ boric acid). Blots were incubated overnight at $4{ }^{\circ} \mathrm{C}$ in $5 \%$ nonfat dry milk in phosphate-buffered saline containing $0.1 \%$ Tween-20 to block unspecific binding. Blots were incubated for $2 \mathrm{~h}$ at room temperature with either mouse monoclonal anti-arginase I (BD Biosciences-Transduction Laboratories, Lexington, KY, USA) (1:1000) or rabbit polyclonal anti-arginase II (Santa Cruz Biotechnology, Santa Cruz, CA, USA) (1:200) antibodies, and for $1 \mathrm{~h}$ with horseradish peroxidase-conjugated antimouse (1:20000) or anti-rabbit (1:10000) IgGs and visualized using enhanced chemiluminescence. Band densities were determined by scanning densitometry. Membranes were stripped and probed with a mouse monoclonal anti- $\beta$-actin antibody (Santa Cruz Biotechnology). Results were expressed as the optical density of the band of interest divided by that for the actin band.

\section{Treatment}

To determine the influence of blood pressure rising by itself on abnormal arginase activity, vasodilator hydralazine hydrochloride (Sigma-Aldrich, SaintQuentin Fallavier, France) was administered $\left(20 \mathrm{mg} \mathrm{kg}^{-1}\right.$ per day in drinking water) for 5 weeks to 5-week-old SHRs. At the end of the hydralazine treatment, measurement of arginase activity was carried out and expression of both isoforms was determined in organs as described above.

\section{Data and statistical analysis}

Values are given as means \pm s.e.m. All data were analyzed by one-way analysis of variance, and statistical significance between the two groups was assessed by Student's unpaired $t$-test. Differences were considered statistically significant when the value was $P<0.05$.

\section{RESULTS}

Body weight, SBP, heart rate, heart and kidney weight indexes The body weight, SBP and heart rate of rats used for the determination of enzyme expression and activity are summarized in Table 1. No difference in body weight was observed between SHRs and WKY rats, except at 26 weeks of age, at which SHRs weighed less than WKY rats $(P<0.05)$. SBP was significantly higher in SHRs than in WKY rats, except at 5 weeks of age, at which time no difference was observed between the two strains. Heart rate was significantly increased in SHRs compared with WKY rats, whatever the age $(P<0.05)$. As shown in Table 1, the kidney mass index was not different between SHRs and WKY rats. By contrast, as a reflection of cardiac hypertrophy, the cardiac mass index was significantly higher in 19- and 26-week-old SHRs compared with that in age-matched WKY rats $(P<0.05)$.

\section{Arginase expression and activity in organs}

As shown in Table 2, in the kidney, liver and brain, arginase activity was not different between WKY rats and SHRs, whatever the age. Similarly, no difference was found in arginase I (liver, brain) or arginase II (kidney, brain) expression between SHRs and WKY rats (Table 3).

Table 1 Characteristics of body weight, systolic blood pressure, heart rate, cardiac and kidney mass index

\begin{tabular}{|c|c|c|c|c|c|}
\hline & $\begin{array}{c}\text { Body } \\
\text { weight, g }\end{array}$ & $\begin{array}{c}S B P, \\
m m H g\end{array}$ & $\begin{array}{c}\text { Heart rate, } \\
\text { beats per min }\end{array}$ & $\begin{array}{c}\text { Kidney mass } \\
\text { index, \% }\end{array}$ & $\begin{array}{c}\text { Cardiac mass } \\
\text { index, } \%\end{array}$ \\
\hline \multicolumn{6}{|c|}{ WKY, weeks } \\
\hline 5 & $134 \pm 2$ & $140 \pm 1$ & $382 \pm 7$ & $0.85 \pm 0.01$ & $0.40 \pm 0.01$ \\
\hline 10 & $227 \pm 4$ & $130 \pm 1$ & $363 \pm 12$ & $0.72 \pm 0.02$ & $0.34 \pm 0.01$ \\
\hline 19 & $342 \pm 4$ & $143 \pm 3$ & $328 \pm 5$ & $0.60 \pm 0.01$ & $0.32 \pm 0.01$ \\
\hline 26 & $381 \pm 12$ & $143 \pm 1$ & $399 \pm 16$ & $0.54 \pm 0.02$ & $0.32 \pm 0.01$ \\
\hline \multicolumn{6}{|c|}{ SHR, weeks } \\
\hline 5 & $134 \pm 2$ & $142 \pm 4$ & $440 \pm 6^{*}$ & $0.84 \pm 0.01$ & $0.42 \pm 0.01$ \\
\hline 10 & $220 \pm 3$ & $186 \pm 5^{*}$ & $407 \pm 11^{*}$ & $0.67 \pm 0.01$ & $0.34 \pm 0.01$ \\
\hline 19 & $336 \pm 6$ & $230 \pm 3^{*}$ & $418 \pm 8^{*}$ & $0.60 \pm 0.01$ & $0.37 \pm 0.01$ * \\
\hline 26 & $371 \pm 9 *$ & $232 \pm 2^{*}$ & $446 \pm 8^{*}$ & $0.59 \pm 0.01$ & $0.41 \pm 0.02 *$ \\
\hline
\end{tabular}

SBP, systolic blood pressure.

Characteristics of body weight, systolic blood pressure, heart rate, cardiac and kidney mass index measured in spontaneously hypertensive rats (SHR) and age-matched Wistar-Kyoto rats (WKY). Results are means \pm s.e.m. from eight rats per group. ${ }^{*}(P<0.05)$ compared with age-matched WKY rats. 
Table 2 Arginase activity in kidney, liver and brain

\begin{tabular}{crrrr}
\hline & \multicolumn{4}{c}{ Arginase activity (pmol urea min ${ }^{-1}$ per mg protein) } \\
\cline { 2 - 5 } & \multicolumn{1}{c}{5 weeks } & 10 weeks & 19 weeks & 26 weeks \\
\hline Kidney & & & & \\
WKY & $999.3 \pm 22.4$ & $1111.9 \pm 38.0$ & $959.1 \pm 36.2$ & $910.7 \pm 35.6$ \\
SHR & $1135.2 \pm 13.9$ & $1107.9 \pm 26.1$ & $1069.3 \pm 63.0$ & $990.9 \pm 25.2$ \\
& & & & \\
Liver & & & & \\
WKY & $871.7 \pm 57.2$ & $885.8 \pm 70.1$ & $1072.5 \pm 37.2$ & $1117.3 \pm 54.9$ \\
SHR & $913.7 \pm 52.5$ & $858.1 \pm 28.0$ & $1172.3 \pm 28.6$ & $1152.3 \pm 35.5$ \\
& & & & \\
Brain & & & & \\
WKY & $922.8 \pm 46.7$ & $939.5 \pm 39.5$ & $1120.6 \pm 38.8$ & $1166.4 \pm 48.5$ \\
SHR & $811.6 \pm 22.5$ & $936.6 \pm 58.4$ & $1120.8 \pm 36.2$ & $1292.1 \pm 65.9$ \\
\hline
\end{tabular}

Arginase activity in kidney, liver and brain in spontaneously hypertensive rats (SHR) and age-matched Wistar-Kyoto rats (WKY). Results are means \pm s.e.m. from eight rats per group.

Table 3 Arginase I and II expression in liver, kidney and brain

\begin{tabular}{|c|c|c|c|c|}
\hline & \multicolumn{4}{|c|}{ Arginase I expression (relative optical density) } \\
\hline & 5 weeks & 10 weeks & 19 weeks & 26 weeks \\
\hline \multicolumn{5}{|l|}{$(A)$} \\
\hline \multicolumn{5}{|l|}{ Liver } \\
\hline WKY & $97 \pm 6$ & $97 \pm 7$ & $96 \pm 6$ & $101 \pm 3$ \\
\hline SHR & $95 \pm 6$ & $92 \pm 8$ & $103 \pm 3$ & $107 \pm 4$ \\
\hline \multicolumn{5}{|l|}{ Brain } \\
\hline WKY & $100 \pm 6$ & $98 \pm 7$ & $97 \pm 6$ & $96 \pm 3$ \\
\hline SHR & $99 \pm 6$ & $94 \pm 8$ & $106 \pm 3$ & $99 \pm 4$ \\
\hline
\end{tabular}

(B)

$\begin{array}{ccccc}\text { Kidney } & & & & \\ \text { WKY } & 93 \pm 6 & 93 \pm 4 & 92 \pm 9 & 82 \pm 5 \\ \text { SHR } & 97 \pm 4 & 97 \pm 7 & 93 \pm 7 & 75 \pm 7 \\ & & & & \\ \text { Brain } & & & & \\ \text { WKY } & 79 \pm 6 & 69 \pm 3 & 65 \pm 6 & 64 \pm 5 \\ \text { SHR } & 67 \pm 6 & 71 \pm 8 & 73 \pm 6 & 69 \pm 8\end{array}$

(A) Arginase I expression in the liver and brain in spontaneously hypertensive rats (SHR) and age-matched Wistar-Kyoto rats (WKY). (B) Arginase II expression in the kidney and brain in spontaneously hypertensive rats (SHR) and age-matched Wistar-Kyoto rats (WKY). Results are means \pm s.e.m. from six rats per group.

By contrast, as shown in Figure 1a, left ventricle arginase activity was higher in hypertensive SHRs aged 10 weeks $(+46 \%, P<0.05), 19$ weeks $(+29 \%, P<0.05)$ and 26 weeks $(+23 \%$, NS) compared with that in age-matched WKY rats. No difference in activity was seen between the two strains at 5 weeks of age. Interestingly, this increased arginase activity was not because of an increased arginase I or II expression, which was not different in left ventricle from SHRs compared with WKY rats (Figures $1 \mathrm{~b}$ and c). Similar results were found in lungs in which arginase activity was increased in SHRs aged 10 weeks $(+39 \%$, $P<0.05)$, 19 weeks $(+49 \%, P<0.05)$ and 26 weeks $(+36 \%, P<0.05)$ compared with WKY rats (Figure $2 \mathrm{a}$ ), without any change in arginase I or II expression (Figures $2 \mathrm{~b}$ and $\mathrm{c}$ ). In addition, in WKY rats, arginase activity increased in an age-dependent manner in the heart but not in the lungs. Thus, compared with 5-week-old WKY rats (Figure 1a), cardiac arginase activity was significantly higher in WKY rats aged 10 weeks $(+14 \%, P<0.05)$, 19 weeks $(+40 \%, P<0.05)$ and 26 weeks $(+54 \%, P<0.05)$. Moreover, cardiac arginase activity was higher in 26-week-old WKY rats than in 10-week-old $(+35 \%$, $P<0.05)$ and 19 -week-old $(+23 \%$, NS, $P=0.1)$ WKY rats.

\section{Effect of hydralazine on arginase activity}

As shown in Figure 3, hydralazine treatment for 5 weeks prevented SBP rising in SHR (Figure 3a). At the end of hydralazine treatment, SBP values in untreated and treated SHRs were $207 \pm 2$ and $166 \pm 3 \mathrm{~mm} \mathrm{Hg}$, respectively $(P<0.05)$. Blood pressure lowering resulted in a decrease in left ventricle arginase activity by $34 \%$ $(P<0.05$, Figure $3 \mathrm{~b})$, whereas it did not affect the lung, liver, kidney and brain arginase activity (data not shown).

\section{DISCUSSION}

Previous data reported that animal models of hypertension are characterized by an increase in arginase activity/expression in conductance and resistance vessels. ${ }^{5,8-11}$ This study investigates for the first time whether abnormalities of the arginase pathway occur outside the vasculature in SHRs, that is, in the heart, lungs, brain, kidney and liver. The results show that (a) tissue arginase activity is not different between prehypertensive SHRs and WKY rats, whatever the organ, (b) hypertensive SHRs exhibit a high arginase activity in the heart and lungs compared with WKY rats, the arginase activity being not impaired in other organs, (c) the prevention of blood pressure rising by hydralazine blunts the increase in arginase activity in the heart but not in the lungs.

Nitric oxide is a cell-cell signaling molecule largely involved in the physiological regulation of many organs, as well as in the pathophysiology of many diseases. The recent demonstration that arginase reciprocally regulates NO levels by competing with NO synthase for their common substrate $\mathrm{L}$-arginine led to a growing interest in the role of this enzyme in many diseases associated with a dysfunction of the Larginine-NO axis, including hypertension. ${ }^{2-6}$ In our study, normotensive WKY rats exhibit substantial arginase activity in all organs studied, which is associated with the expression of both arginase isoforms in the brain, lungs and heart, and with the expression of arginase I in the liver and arginase II in the kidney. These data are in accordance with previous studies reporting the expression of arginase I and/or II in neurons, ${ }^{16,17}$ cardiomyocytes, ${ }^{18,19}$ pulmonary epithelial, endothelial, smooth muscle cells, fibroblasts and alveolar macrophages ${ }^{20}$ in renal proximal straight tubules and inner medullary collecting ducts, ${ }^{21,22}$ as well as in hepatocytes. ${ }^{21} \mathrm{~A}$ new finding of our study is the age-dependent increase in arginase activity in the heart of WKY rats. These data suggest that cardiac arginase is a target of age-associated metabolic dysfunctions and complete recent studies showing increased vascular ${ }^{23}$ and penile ${ }^{24}$ arginase in old animals. Given that aging is associated with impaired myocardial contractility ${ }^{25}$ and that arginase activity was reported to modulate cardiomyocyte contractility through an NO-dependent mechanism, ${ }^{18,19}$ this result opens new avenues on the role of arginase in cardiac dysfunction associated with aging.

Hypertension is a major risk for cardiovascular mortality and morbidity through its effects on target organs such as the brain, heart and kidney. Subtle target-organ damage such as left-ventricular hypertrophy, microalbuminuria and cognitive dysfunction takes place early in the course of hypertensive cardiovascular diseases, although catastrophic events such as stroke, heart attack, renal failure and 


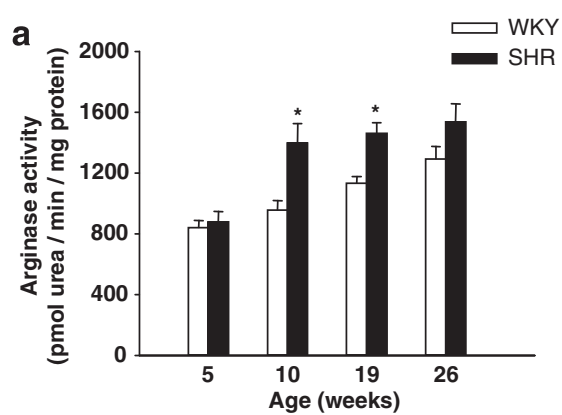

b

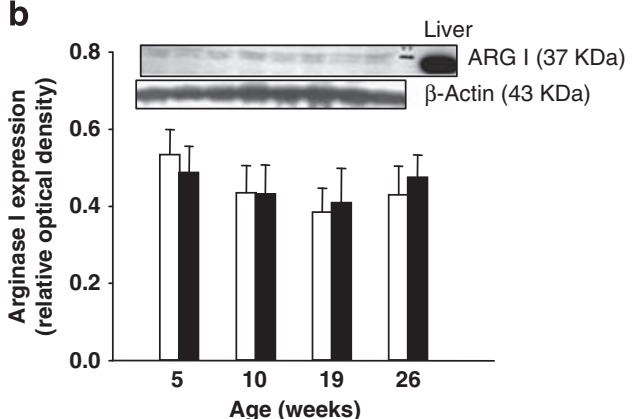

C

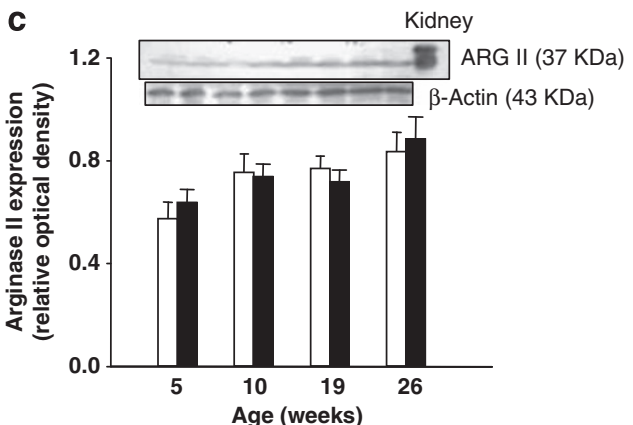

Figure 1 Arginase activity (a) and expression ( $\mathbf{b}$ and $\mathbf{c}$ ) in the left ventricle of spontaneously hypertensive rats (SHRs) and of age-matched Wistar-Kyoto rats (WKY). Results are means \pm s.e.m. from eight rats per group. ${ }^{*}(P<0.05)$ versus that of WKY rats. ARG, arginase.
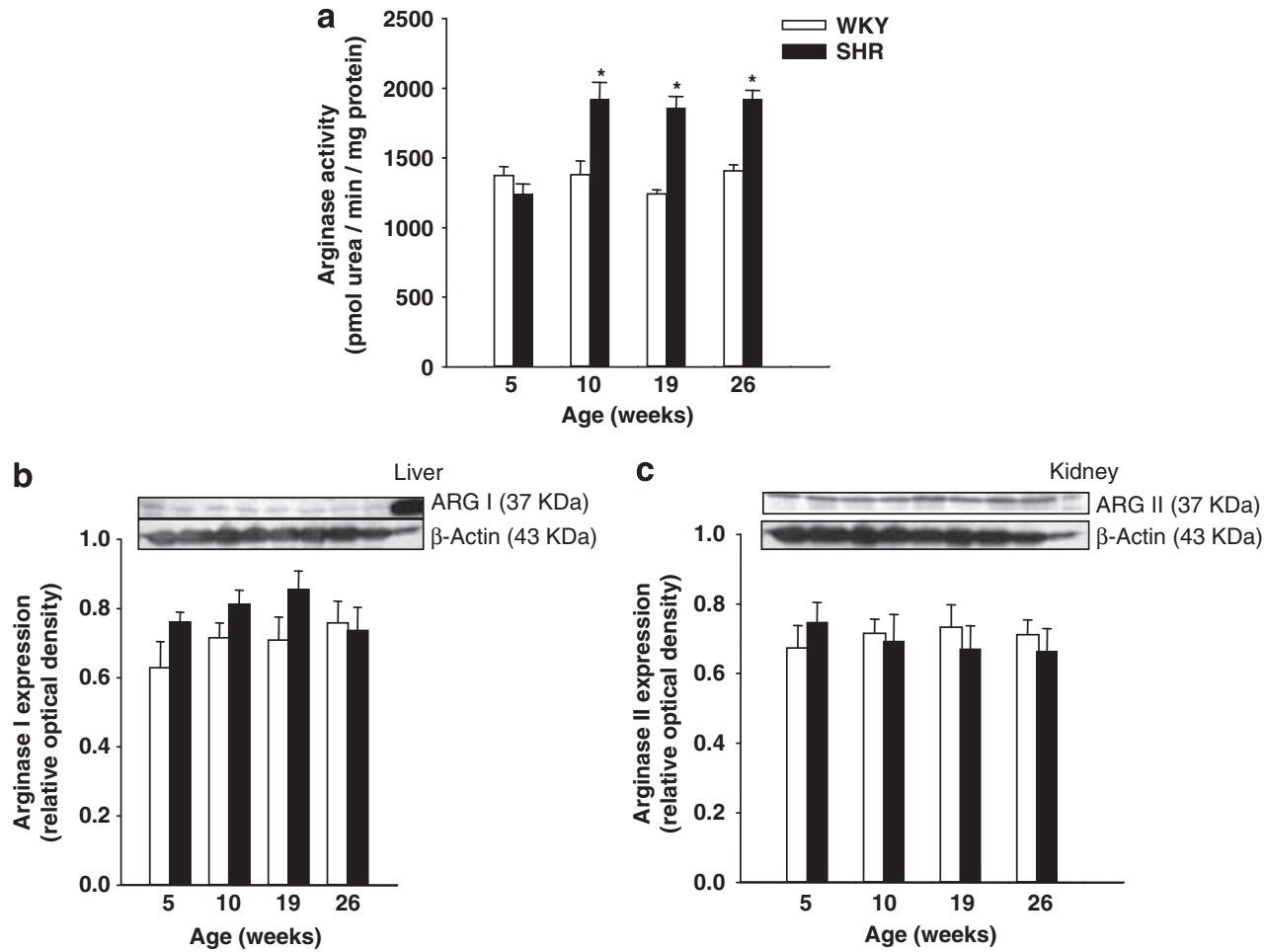

Figure 2 Arginase activity (a) and expression in the lungs of spontaneously hypertensive rats (SHRs) and of age-matched Wistar-Kyoto rats (WKY). (a) Arginase activity was determined in pulmonary tissue of SHRs and WKY rats from urea production using the spectrophotometric method of Corraliza et al. ${ }^{13}$ Results are means \pm s.e.m. from eight rats per group. (b) Pulmonary arginase I and (c) arginase II expressions were determined by western blot. Total proteins were separated, and analysis was performed using the monoclonal anti-arginase I (b) or polyclonal anti-arginase II (c) antibodies as described in Methods. The upper panels show representative immunoblots and lower panels illustrate densitometric analysis of protein levels. Results are means \pm s.e.m. from six rats per group. ${ }^{*}(P<0.05)$ versus that of WKY rats. ARG, arginase.

dementia usually happen after long periods of uncontrolled hypertension only. ${ }^{26}$ Hypertensive end-organ damage likely relies on structural and functional changes in vasculature, including arteriolar remodeling and capillary rarefaction. ${ }^{26}$ To date, whether the vascular arginase upregulation reported in models of secondary or genetic hypertension $^{5,8-10}$ accounts for the development of target organ damage is not 

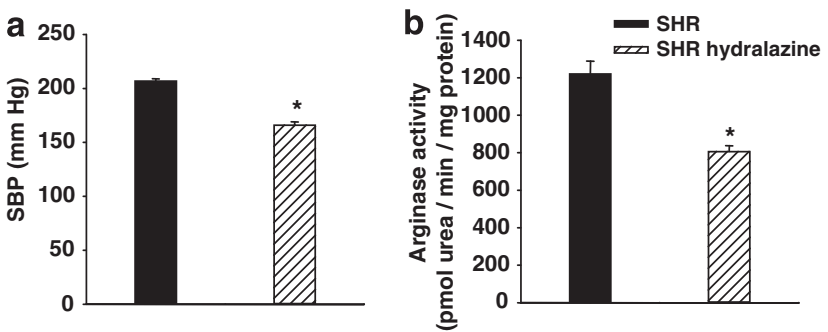

Figure 3 Effect of hydralazine on systolic blood pressure (SBP) and arginase activity in cardiac tissue from spontaneously hypertensive rats (SHRs). (a) Blood pressure was measured in 10-week-old SHRs treated with or without hydralazine $\left(20 \mathrm{mg} \mathrm{kg}^{-1}\right.$ per day) in drinking water for 5 weeks. (b) Arginase activity was determined in cardiac tissue of SHRs from urea production using the spectrophotometric method of Corraliza et al. ${ }^{13}$ Results are means \pm s.e.m. from eight rats per group. ${ }^{*}(P<0.05)$ versus that of SHRs.

known. In this study, we measured arginase activity in whole organs, that is, in homogenates containing parenchyma, stroma and vascular cells. With regard to the low level of arginase expression in vessels and stroma cells, compared with that in parenchyma cells, ${ }^{27}$ arginase activity measured in whole organ likely reflects the activity in parenchyma cells. Our results report an increased arginase activity in the heart but not in the brain or in the kidney from hypertensive SHRs. These data suggest that hypertension-induced damage to the heart is associated with changes in arginase activity not only in coronary arterioles, as previously reported in pigs with aortic coarctation, ${ }^{5}$ but also in extravascular cardiac components. They also suggest that the misregulation of the arginase pathway in cardiac cells might contribute to hypertension-induced cardiac hypertrophy. Further experiments are needed to confirm this hypothesis. By contrast, the lack of changes in arginase activity in the brain and kidneys of SHRs does not support a role of extravascular arginase in hypertensioninduced renal and cognitive dysfunction. In accordance with this hypothesis are the discrepancies observed between our data on renal arginase activity in SHRs and previous data obtained on secondary models of hypertension. In these studies, a decreased arginase activity was measured in kidneys from Dahl/Rapp salt-sensitive rats ${ }^{28}$ and deoxycortisone acetate-salt hypertensive rats. ${ }^{8}$ Given that these three models of hypertension are associated with renal dysfunction, ${ }^{29}$ these conflicting data on renal arginase activity do not argue for an important role of parenchymal arginase in hypertension-induced nephropathy.

Lungs - which are not exposed to systemic blood pressure-are not considered as 'typical' target organs of hypertension. However, growing evidence indicates that hypertensive SHRs exhibit lung abnormalities such as inflammation, hemorrhage, oxidative burden and increased vascular leakage, ${ }^{30}$ and that SHRs are more sensitive to air pollutants $^{31,32}$ and bronchoconstrictive agents than are WKY rats. ${ }^{33}$ Although the physiological function of arginase in lungs is not known, ${ }^{34}$ previous studies showed that increased arginase activity contributes to the airway hyperresponsiveness associated with asthma and allergy airway diseases by likely reducing the bioavailability of L-arginine for NO synthases. ${ }^{34}$ Therefore, our results that report an increased arginase activity in the lungs of SHRs might provide a new potential mechanism involved in the susceptibility of SHRs to pulmonary complications. Further experiments are needed to clarify this point.

Both translational and posttranslational mechanisms may theoretically be involved in increased arginase activity in the heart and lungs of hypertensive SHRs. However, our results that report changes in arginase activity without concomitant changes in arginase expression support the hypothesis that hypertension selectively targets posttranslational mechanisms in these organs. The fact that the lung and heart arginase activity upregulation was not observed in SHRs at the prehypertensive stage but only after the development of hypertension strongly suggests that increased arginase activity is related to blood pressure elevation. To test this hypothesis, we measured arginase activity in SHRs receiving an antihypertensive therapy with hydralazine, a direct acting vasodilator that reduces peripheral resistance by relaxing the smooth muscle cell layer in arterial vessels. ${ }^{35}$ Our data show that preventing a rise in blood pressure blunts the increase in arginase activity in the heart but not in the lungs. Unlike the lungs, myocardial tissue is directly submitted to high-pressure overload secondary to increased systemic blood pressure. Thus, the effect of hydralazine on the heart argues for a hemodynamic sensitivity of cardiac arginase, as previously shown in aortas from SHRs. ${ }^{11}$ However, besides its antihypertensive properties, hydralazine is also an inhibitor of NADH oxidase activity. ${ }^{36}$ Given that SHR exhibits increased oxidative stress in the heart, ${ }^{37}$ and that recent evidence suggests that arginase activity increases in response to oxidative stress, ${ }^{38,39}$ the possibility that hydralazine decreased arginase activity by an antioxidant rather than by a pressure-dependent mechanism cannot be ruled out. However, if true, lung arginase activity, which seems to be independent of blood pressure values, should have been reduced by hydralazine, as oxidative stress is also evident in the lungs of SHRs. ${ }^{30}$ In previous studies conducted on cultured vascular cells or vessels, a high RhoA activity, ${ }^{40}$ microtubule depolymerization, ${ }^{41}$ decreased production of the endogenous arginase inhibitor $N^{\mathrm{G}}$-hydroxy-Larginine (L-NOHA) ${ }^{42}$ and S-nitrosylation of arginase by inducible $\mathrm{NOS}^{23}$ have been shown to increase arginase activity independently of increased arginase expression. Whether these mechanisms might contribute to an increased arginase activity in the lungs remains to be determined.

In conclusion, our results show that increased arginase activity is not confined to large vessels in genetic hypertension but also occurs in the heart and lungs, at least in hypertensive stages. They also give arguments for the hemodynamic sensitivity of cardiac but not lung arginase activity in essential hypertension. These findings open a new field of investigation aimed at understanding the functional consequences of abnormal arginase activity in the heart and lungs in genetic hypertension.

\section{ACKNOWLEDGEMENTS}

This work was supported by a grant from the Société Française d'Hypertension Artérielle.

1 Jenkinson CP, Grody WW, Cederbaum SD. Comparative properties of arginases. Comp Biochem Physiol B Biochem Mol Biol 1996; 114: 107-132.

2 Buga GM, Singh R, Pervin S, Rogers NE, Schmitz DA, Jenkinson CP, Cederbaum SD, Ignarro LJ. Arginase activity in endothelial cells: inhibition by NG-hydroxy-L-arginine during high-output NO production. Am J Physiol 1996; 271: H1988-H1998.

3 Ignarro LJ, Buga GM, Wei LH, Bauer PM, Wu G, del Soldato P. Role of the arginine-nitric oxide pathway in the regulation of vascular smooth muscle cell proliferation. Proc Natl Acad Sci USA 2001; 98: 4202-4208.

4 Zhang C, Hein TW, Wang W, Chang Cl, Kuo L. Constitutive expression of arginase in microvascular endothelial cells counteracts nitric oxide-mediated vasodilatory function. Faseb J 2001; 15: 1264-1266.

5 Zhang C, Hein TW, Wang W, Miller MW, Fossum TW, McDonald MM, Humphrey JD, Kuo L. Upregulation of vascular arginase in hypertension decreases nitric oxidemediated dilation of coronary arterioles. Hypertension 2004; 44: 935-943.

6 Berkowitz DE, White R, Li D, Minhas KM, Cernetich A, Kim S, Burke S, Shoukas AA, Nyhan D, Champion HC, Hare JM. Arginase reciprocally regulates nitric oxide synthase 
activity and contributes to endothelial dysfunction in aging blood vessels. Circulation 2003; 108: 2000-2006.

7 Landmesser U, Drexler H. Endothelial function and hypertension. Curr Opin Cardiol 2007; 22: 316-320.

8 Rodriguez S, Richert L, Berthelot A. Increased arginase activity in aorta of mineralocorticoid-salt hypertensive rats. Clin Exp Hypertens 2000; 22: 75-85.

9 Johnson FK, Johnson RA, Peyton KJ, Durante W. Arginase inhibition restores arteriolar endothelial function in Dahl rats with salt-induced hypertension. Am J Physiol Regul Integr Comp Physiol 2005; 288: R1057-R1062.

10 Demougeot C, Prigent-Tessier A, Marie C, Berthelot A. Arginase inhibition reduces endothelial dysfunction and blood pressure rising in spontaneously hypertensive rats. $J$ Hypertens 2005; 23: 971-978.

11 Demougeot C, Prigent-Tessier A, Bagnost T, Andre C, Guillaume Y, Bouhaddi M, Marie C, Berthelot A. Time course of vascular arginase expression and activity in spontaneously hypertensive rats. Life Sci 2007; 80: 1128-1134.

12 Bagnost T, Berthelot A, Bouhaddi M, Laurant P, Andre C, Guillaume Y, Demougeot C. Treatment with the arginase inhibitor N(omega)-hydroxy-nor-L-arginine improves vascular function and lowers blood pressure in adult spontaneously hypertensive rat. $J$ Hypertens 2008; 26: 1110-1118.

13 Corraliza IM, Campo ML, Soler G, Modolell M. Determination of arginase activity in macrophages: a micromethod. J Immunol Meth 1994; 174: 231-235.

14 Lowry OH, Rosebrough NJ, Farr AL, Randall RJ. Protein measurement with the Folin phenol reagent. J Biol Chem 1951; 193: 265-275.

15 Laemmli UK. Cleavage of structural proteins during the assembly of the head of bacteriophage T4. Nature 1970; 227: 680-685.

16 Yu H, lyer RK, Kern RM, Rodriguez WI, Grody WW, Cederbaum SD. Expression of arginase isozymes in mouse brain. J Neurosci Res 2001; 66: 406-422.

17 Liu P, Smith PF, Appleton I, Darlington CL, Bilkey DK. Regional variations and agerelated changes in nitric oxide synthase and arginase in the sub-regions of the hippocampus. Neuroscience 2003; 119: 679-687.

18 Steppan J, Ryoo S, Schuleri KH, Gregg C, Hasan RK, White AR, Bugaj LJ, Khan M, Santhanam L, Nyhan D, Shoukas AA, Hare JM, Berkowitz DE. Arginase modulates myocardial contractility by a nitric oxide synthase 1-dependent mechanism. Proc Natl Acad Sci USA 2006; 103: 4759-4764.

19 Jung AS, Kubo H, Wilson R, Houser SR, Margulies KB. Modulation of contractility by myocyte-derived arginase in normal and hypertrophied feline myocardium. Am J Physiol Heart Circ Physiol 2006; 290: H1756-H1762.

20 Maarsingh H, Pera T, Meurs H. Arginase and pulmonary diseases. Naunyn Schmiedebergs Arch Pharmacol 2008; 378: 171-184.

21 Miyanaka K, Gotoh T, Nagasaki A, Takeya M, Ozaki M, Iwase K, Takiguchi M, Iyama KI, Tomita K, Mori M. Immunohistochemical localization of arginase II and other enzymes of arginine metabolism in rat kidney and liver. Histochem J 1998; 30: 741-751.

22 Levillain O, Balvay S, Peyrol S. Mitochondrial expression of arginase II in male and female rat inner medullary collecting ducts. J Histochem Cytochem 2005; 53: 533-541.

23 Santhanam L, Lim HK, Miriel V, Brown T, Patel M, Balanson S, Ryoo S, Anderson M, Irani K, Khanday F, Di Costanzo L, Nyhan D, Hare JM, Christianson DW, Rivers R, Shoukas A, Berkowitz DE. Inducible NO synthase dependent S-nitrosylation and activation of arginase-1 contribute to age-related endothelial dysfunction. Circ Res 2007; 101: 692-702.

24 Santhanam L, Christianson DW, Nyhan D, Berkowitz DE. Arginase and vascular aging. J App/ Physiol 2008; 105: 1632-1642.
25 Lakatta EG. Cardiovascular ageing in health sets the stage for cardiovascular disease. Heart Lung Circ 2002; 11: 76-91.

26 Cohuet G, Struijker-Boudier H. Mechanisms of target organ damage caused by hypertension: therapeutic potential. Pharmacol Ther 2006; 111: 81-98.

27 Belik J, Shehnaz D, Pan J, Grasemann H. Developmental changes in arginase expression and activity in the lung. Am J Physiol Lung Cell Mol Physiol 2008; 294: L498-L504.

28 Iwata S, Tsujino T, Ikeda Y, Ishida T, Ueyama T, Gotoh T, Mori M, Yokoyama M. Decreased expression of arginase II in the kidneys of Dahl salt-sensitive rats. Hypertens Res 2002; 25: 411-418.

29 Pinto YM, Paul M, Ganten D. Lessons from rat models of hypertension: from Goldblatt to genetic engineering. Cardiovasc Res 1998; 39: 77-88.

30 Kodavanti UP, Schladweiler MC, Ledbetter AD, Ortuno RV, Suffia M, Evansky $P$, Richards JH, Jaskot RH, Thomas R, Karoly E, Huang YC, Costa DL, Gilmour PS, Pinkerton KE. The spontaneously hypertensive rat: an experimental model of sulfur dioxide-induced airways disease. Toxicol Sci 2006; 94: 193-205.

31 Cao Q, Zhang S, Dong C, Song W. Pulmonary responses to fine particles: differences between the spontaneously hypertensive rats and Wistar Kyoto rats. Toxicol Lett 2007; 171: 126-137.

32 Yu B, Kodavanti UP, Takeuchi M, Witschi H, Pinkerton KE. Acute tobacco smokeinduced airways inflammation in spontaneously hypertensive rats. Inhal Toxicol 2008; 20: 623-633.

33 Kwasniewski FH, Landgraf RG, Bakhle YS, Jancar S. Bronchoconstriction and endogenous nitric oxide in isolated lungs of spontaneously hypertensive rats. Eur $J$ Pharmacol 2004; 488: 181-189.

34 Maarsingh $\mathrm{H}$, Zaagsma J, Meurs $\mathrm{H}$. Arginine homeostasis in allergic asthma. Eur J Pharmacol 2008; 585: 375-384.

35 Ellershaw DC, Gurney AM. Mechanisms of hydralazine induced vasodilation in rabbit aorta and pulmonary artery. Br J Pharmacol 2001; 134: 621-631.

36 Munzel T, Kurz S, Rajagopalan S, Thoenes M, Berrington WR, Thompson JA, Freeman BA, Harrison DG. Hydralazine prevents nitroglycerin tolerance by inhibiting activation of a membrane-bound NADH oxidase. A new action for an old drug. J Clin Invest 1996; 98: 1465-1470.

37 Alvarez MC, Caldiz C, Fantinelli JC, Garciarena CD, Console GM, Chiappe de Cingolani GE, Mosca SM. Is cardiac hypertrophy in spontaneously hypertensive rats the cause or the consequence of oxidative stress? Hypertens Res 2008; 31: 1465-1476.

38 Iyamu EW, Perdew H, Woods GM. Cysteine-iron promotes arginase activity by driving the Fenton reaction. Biochem Biophys Res Commun. 2008; 376: 116-120.

39 Thengchaisri N, Hein TW, Wang W, Xu X, Li Z, Fossum TW, Kuo L. Upregulation of arginase by $\mathrm{H} 2 \mathrm{O} 2$ impairs endothelium-dependent nitric oxide-mediated dilation of coronary arterioles. Arterioscler Thromb Vasc Biol 2006; 26: 2035-2042.

40 Ming XF, Barandier C, Viswambharan H, Kwak BR, Mach F, Mazzolai L, Hayoz D, Ruffieux J, Rusconi S, Montani JP, Yang Z. Thrombin stimulates human endothelial arginase enzymatic activity via RhoA/ROCK pathway: implications for atherosclerotic endothelial dysfunction. Circulation 2004; 110: 3708-3714.

41 Ryoo S, Lemmon CA, Soucy KG, Gupta G, White AR, Nyhan D, Shoukas A, Romer LH, Berkowitz DE. Oxidized low-density lipoprotein-dependent endothelial arginase II activation contributes to impaired nitric oxide signaling. Circ Res 2006: 99: 951-960.

42 Masuda $\mathrm{H}$. Significance of nitric oxide and its modulation mechanisms by endogenous nitric oxide synthase inhibitors and arginase in the micturition disorders and erectile dysfunction. Int J Urol 2008; 15: 128-134. 